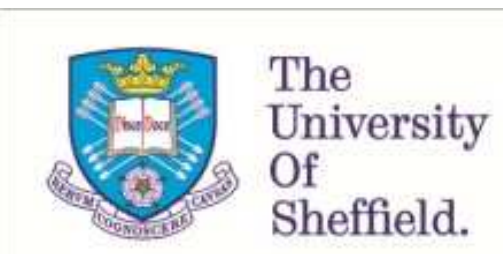

This is a repository copy of Quasi-invariance for the Navier-Stokes equations.

White Rose Research Online URL for this paper:

http://eprints.whiterose.ac.uk/142435/

Version: Accepted Version

\title{
Book Section:
}

Ohkitani, K. (2018) Quasi-invariance for the Navier-Stokes equations. In: Fefferman, C.L., Robinson, J.C. and Rodrigo, J.L., (eds.) Partial Differential Equations in Fluid Mechanics. London Mathematical Society Lecture Note Series, 452 . Cambridge University Press, Cambridge , pp. 97-112. ISBN 9781108460965

This article has been published in a revised form in Fefferman, C. et al (eds.) Partial Differential Equations in Fluid Mechanics. This version is free to view and download for private research and study only. Not for re-distribution, re-sale or use in derivative works. (c) Cambridge University Press.

\section{Reuse}

Items deposited in White Rose Research Online are protected by copyright, with all rights reserved unless indicated otherwise. They may be downloaded and/or printed for private study, or other acts as permitted by national copyright laws. The publisher or other rights holders may allow further reproduction and re-use of the full text version. This is indicated by the licence information on the White Rose Research Online record for the item.

\section{Takedown}

If you consider content in White Rose Research Online to be in breach of UK law, please notify us by emailing eprints@whiterose.ac.uk including the URL of the record and the reason for the withdrawal request. 


\title{
Quasi-invariance for the Navier-Stokes equations
}

\author{
Koji Ohkitani \\ School of Mathematics and Statistics \\ The University of Sheffield \\ Hicks Building, Hounsfield Road \\ Sheffield S3 7RH, U.K.
}




\title{
Quasi-invariance for the Navier-Stokes equations
}

\begin{abstract}
In this contribution we focus on a few results regarding the study of the three-dimensional Navier-Stokes equations with use of the vector potentials. These dependent variables are critical in the sense that they are scale-invariant. By surveying recent results utilising criticality of various norms, we emphasise the advantages of working with scaleinvariant variables.

The Navier-Stokes equations, which are invariant under static scaling transforms, are not invariant under dynamic scaling transforms. Using the vector potential, we introduce scale-invariance in a weaker form, that is, invariance under dynamic scaling modulo a martingale (Maruyama-Girsanov density) when the equations are cast into Wiener path-integrals. We briefly discuss the implications of this quasi-invariance on the basic issues of the Navier-Stokes equations.
\end{abstract}

\subsection{Introduction}

Many of the results in Navier-Stokes theory have been obtained by paying attention to scale-invariant properties of norms, stemming from e.g. Kato \& Fujita (1962). Recent researches in this line include: exclusion of self-similar blowup in Nečas, Růžička \& Šverák (1996) also Chae (2007); Hou \& Li (2007), the regularity criterion by the $L^{3}$-norm of velocity in Escauriaza, Seregin \& Sverak (2003) and the global regularity result for small initial data in $\mathrm{BMO}^{-1}$-norm in Koch \& Tataru (2001). These results are motivated, at least partly, by static (i.e. frozen time) scale-invariant considerations of the Navier-Stokes equations under the usual parabolic transformations.

There is more general, yet another kind of transformations, which is 
dynamical in nature. In view of these successful results with static scaling transformations, it seems promising to pursue further development with dynamic scaling transformations. Through such efforts, it has been recognised recently that with the scale-invariant dependent variables we would benefit from some advantages in the analysis. In particular, we can characterise the concept of scale-invariance in its most generalised sense. The purpose of this contribution is to survey a number of results on the basic problems of the Navier-Stokes equations obtained in this spirit. This survey is not intended to be an exhaustive list of literature, but it is rather an idiosyncratic review.

The rest of this paper is constructed as follows. In section 2, we describe the usual reformulation of the Navier-Stokes equations as integral equations using the velocity variable. In section 3 , we recall how we may solve the forced Burgers equations by linearisation and a path-integral. In Section 4, we recall the Navier-Stokes equations written in the vector potentials and some regularity conditions using critical and subcritical norms. Section 5, we apply an analogue of the Cole-Hopf transform and the Feynman-Kac formula just as we did for the Burgers equations. In Section 6 , by dynamic scaling transform we derive the Leray equations. Using probabilistic tools we compare the Navier-Stokes and Leray equations in detail, thereby recognising their quasi-invariance. Section 7 is devoted to a summary.

\subsection{Navier-Stokes equations}

We are interested in the Navier-Stokes equations in $\mathbb{R}^{3}$ :

$$
\begin{array}{r}
\frac{\partial \boldsymbol{u}}{\partial t}+\boldsymbol{u} \cdot \nabla \boldsymbol{u}=-\nabla p+\frac{1}{2} \triangle \boldsymbol{u}, \\
\nabla \cdot \boldsymbol{u}=0, \\
\boldsymbol{u}(\boldsymbol{x}, 0)=\boldsymbol{u}_{0}(\boldsymbol{x}) .
\end{array}
$$

Starting from Leray (1934), lots of efforts have been made on the analyses of the Navier-Stokes equations. General references include Constantin \& Foias (1988); Doering (2009); Doering \& Gibbon (1995); Robinson \& Sadowski (2009).

It is useful to recall how we can convert the above equations to the conventional integral equations. Using the heat kernel

$$
g_{t}=\frac{1}{(2 \pi t)^{3 / 2}} \exp \left(-\frac{|\boldsymbol{x}|^{2}}{2 t}\right),
$$


we apply the Duhamel principle to the Navier-Stokes equations to obtain

$$
\begin{aligned}
& \boldsymbol{u}(t)=g_{t} * \boldsymbol{u}_{0}-\int_{0}^{t} g_{t-s} * \mathbb{P} \nabla \cdot(\boldsymbol{u} \otimes \boldsymbol{u})(\cdot, s) d s, \\
= & \mathbb{E}\left[\boldsymbol{u}_{0}\left(\boldsymbol{W}_{t}\right)\right]-\int_{0}^{t} \mathbb{E}\left[\mathbb{P} \nabla \cdot(\boldsymbol{u} \otimes \boldsymbol{u})\left(\boldsymbol{W}_{s}, t-s\right)\right] d s
\end{aligned}
$$

where $\mathbb{P}=\boldsymbol{I}-\nabla \triangle^{-1} \nabla$. denotes solenoidal projection and $*$ convolution. Here $\boldsymbol{W}_{t}$ denotes three-dimensional standard Brownian motion starting from $\boldsymbol{x}$ at $t=0 \boldsymbol{W}_{0}=\boldsymbol{x}$ and $\mathbb{E}[\cdot]$ an average with respect to a probability measure associated with $\boldsymbol{W}_{t}$. See Appendix A.

The following condition due to Serrin (1963)

$$
\int_{0}^{T}\|\boldsymbol{u}\|_{L^{p}}^{\frac{2 p}{p-3}} d t<\infty, \quad(3<p \leq \infty)
$$

guarantees uniqueness and smoothness of the solution on $[0, T)$. In particular, we have in the limit $p \rightarrow \infty$

$$
\int_{0}^{T}\|\boldsymbol{u}\|_{L^{\infty}}^{2} d t<\infty
$$

which is probably the best-known criterion for regularity. Different arguments - based on different ways to estimate the right-hand side of (1.2) - are required to obtain the two regularity criteria in (1.3) and (1.4) (see the contribution by Ozanski \& Pooley in this volume). The condition (1.4) can be compared with (1.17) in the equation (1.15) below, which is obtained as a boundedness condition for the exponential process to be a martingale. This illustratesan advantage of working with critical dependent variables.

\subsection{Burgers equation}

To illustrate the basic ideas, we consider the Burgers equations in $\mathbb{R}^{3}$ subject to an external forcing of the form $-\nabla V(\boldsymbol{x}, t)$

$$
\begin{array}{r}
\frac{\partial \boldsymbol{v}}{\partial t}+\boldsymbol{v} \cdot \nabla \boldsymbol{v}=-\nabla V+\frac{1}{2} \triangle \boldsymbol{v}, \\
\boldsymbol{v}(\boldsymbol{x}, 0)=\boldsymbol{v}_{0}(\boldsymbol{x}) .
\end{array}
$$

(More generally, the following argument holds in any $\mathbb{R}^{n}, n \geq 1$.) Here we restrict ourselves to the special class of potential flows $\boldsymbol{v}=\nabla \phi$. The variable $\boldsymbol{v}$ satisfies the following well-known scale-invariance: 
if $\boldsymbol{v}(\boldsymbol{x}, t)$ is a solution, then so is $\lambda \boldsymbol{v}\left(\lambda \boldsymbol{x}, \lambda^{2} t\right)$ for any $\lambda>0$.

Integrating the equation (1.5) and taking the constant of integration to be zero, we find the following Hamilton-Jacobi equation

$$
\begin{aligned}
\frac{\partial \phi}{\partial t}+\frac{1}{2}|\nabla \phi|^{2}+V & =\frac{1}{2} \Delta \phi, \\
\phi(\boldsymbol{x}, 0) & =\phi_{0}(\boldsymbol{x}) .
\end{aligned}
$$

In terms of the variable $\phi$, scale-invariance now reads

$$
\text { if } \phi(\boldsymbol{x}, t) \text { is a solution, then so is } \phi\left(\lambda \boldsymbol{x}, \lambda^{2} t\right) \text { for any } \lambda>0 \text {. }
$$

An observation made by Cole $(1949,1951)$ is that $\phi$ lacks a prefactor after the transformation. This is because $\phi$ has the same physical dimension as kinematic viscosity $\nu(=1 / 2)$. Applying a transform $\phi=k \log \theta$, with a constant $k$ of the same dimension as $\nu$, we rewrite (1.6) as

$$
\begin{array}{r}
\frac{\partial \theta}{\partial t}=\frac{1}{2} \Delta \theta-\left(\frac{k+1}{2} \frac{|\nabla \theta|^{2}}{\theta^{2}}+\frac{V}{k}\right) \theta, \\
\theta(\boldsymbol{x}, 0)=\theta_{0}(\boldsymbol{x}) .
\end{array}
$$

Choosing $k=-1$ and following Cole (1951); Hopf (1950), we can linearise the Burgers equation to a heat equation with a potential term, i.e. the Schrödinger equation at imaginary times

$$
\frac{\partial \theta}{\partial t}=\frac{1}{2} \triangle \theta+V \theta \text {. }
$$

If the potential term is bounded in the sense that

$$
\int_{0}^{t} \sup _{\boldsymbol{x}}|V(\boldsymbol{x}, s)| d s<\infty
$$

the equation (1.7) is soluble by the Feynman-Kac formula as

$$
\theta(\boldsymbol{x}, t)=E\left[\theta_{0}\left(\boldsymbol{W}_{t}\right) \exp \left(\int_{0}^{t} V\left(\boldsymbol{W}_{s}, s\right) d s\right)\right] .
$$

This representation can be obtained by applying a time-dependent Trotter formula, see e.g. Taylor (1996). See Appendix B for alternative forms of functional integrals. Note that solutions $\theta_{k}$ for $k \neq-1$ can be obtained as $\theta_{k}=\theta^{-1 / k}$.

We refer (1.8) to the Cole-Hopf-Feynman-Kac formula for the Burgers equations. We will take a brute-force approach to obtain a similar expression for the Navier-Stokes equations. 


\subsection{Use of critical dependent variables}

\begin{tabular}{cccccc} 
& $\mathrm{BMO}^{-1}$ & $L^{3}$ & $\dot{H}^{1 / 2}$ & $L^{p}(p>3)$ & $\dot{H}^{1}$ \\
\hline $\begin{array}{c}\text { small data } \rightarrow \\
\text { global regularity }\end{array}$ & $\begin{array}{c}\text { Yes } \\
\mathrm{KT}(' 01)\end{array}$ & Yes & $\begin{array}{c}\text { Yes } \\
\mathrm{KF}(' 62)\end{array}$ & NA & NA \\
\hline $\begin{array}{c}\text { time of } \\
\text { local existence }\end{array}$ & $\mathrm{NA}$ & $\mathrm{NA}$ & $\mathrm{NA}$ & $\left\|\boldsymbol{u}_{0}\right\|^{-\frac{2 p}{p-3}}$ & $\nu^{3} /\left\|\boldsymbol{u}_{0}\right\|_{H^{1}}^{4}$ \\
\hline blowup criterion & Unknown & Yes & Yes & Yes & Yes
\end{tabular}

Table 1: Here $\boldsymbol{u}_{0}$ denotes the initial velocity and $\|\boldsymbol{u}\|_{\mathrm{BMO}^{-1}} \approx\|\boldsymbol{\psi}\|_{\mathrm{BMO}}$. Note that NA's appear in a staggered manner; for the three critical norms (on the left) and the two subcritical norms (on the right). KT('01) refers to Koch \& Tataru (2001) and KF('62) to Kato \& Fujita (1962).

We introduce the vector potentials $\boldsymbol{\psi}$ defined in such a way that $\boldsymbol{u}=$ $\nabla \times \boldsymbol{\psi}$ and $\nabla \cdot \boldsymbol{\psi}=0$. The Navier-Stokes equations have been written as a nonlocal version of the Hamilton-Jacobi equations in Ohkitani (2015)

$$
\frac{\partial \boldsymbol{\psi}}{\partial t}-\frac{1}{2} \triangle \boldsymbol{\psi}=\boldsymbol{T}[\nabla \boldsymbol{\psi}]
$$

where

$$
\boldsymbol{T}[\nabla \boldsymbol{\psi}] \equiv \frac{3}{4 \pi} \int_{\mathbb{R}^{3}} \frac{\boldsymbol{r} \times(\nabla \times \boldsymbol{\psi}(\boldsymbol{y})) \boldsymbol{r} \cdot(\nabla \times \boldsymbol{\psi}(\boldsymbol{y}))}{|\boldsymbol{r}|^{5}} \mathrm{~d} \boldsymbol{y},
$$

with $\boldsymbol{r}=\boldsymbol{x}-\boldsymbol{y}$ and $f$ denotes a principal-value integral. We assume that $|\boldsymbol{\psi}(\boldsymbol{x}, t)| \rightarrow 0$ as $|\boldsymbol{x}| \rightarrow \infty$ for all $t \geq 0$. It can be checked that $\nabla \cdot \boldsymbol{T}[\nabla \boldsymbol{\psi}]=0$ is satisfied.

In Table 1 we compare a number of known results on the NavierStokes regularity. One kind of theorems claims global regularity for small initial data, while the other kind local existence for general initial data. We list results obtained with critical $\mathrm{BMO}^{-1}, L^{3}$ and $\dot{H}^{1 / 2}$-norms and those with subcritical $L^{p}(p>3)$ and $\dot{H}^{1}$-norms. If any one of NA's were available, that would imply global regularity immediately. See also Ohkitani (2016) for an asymptotic analysis related to $\dot{H}^{1 / 2}$-norm.

Experience shows that those two kinds of theorems go together; in view of the embedding

$$
\|\boldsymbol{\psi}\|_{\mathrm{BMO}} \lesssim\|\boldsymbol{u}\|_{L^{3}}
$$

we may ask whether

$$
\|\psi\|_{\mathrm{BMO}} \rightarrow \infty \text { as } t \rightarrow t_{*}
$$


for a possible blowup at $t_{*}$. Apparently, this remains an open question (hence a question mark in Table 1) and we will briefly remark on it, in connection with the Cole-Hopf transform at the end of Section 5.

At the moment, it is known from Ohkitani (2017a) that the following condition

$$
\int_{0}^{t_{*}}\|\boldsymbol{T}[\nabla \boldsymbol{\psi}]\|_{L^{\infty}} d t=\infty
$$

holds for blowup at $t=t_{*}$.

A possibility of an even weaker norm $\|\boldsymbol{u}\|_{B_{\infty, \infty}^{-1}}$ serving as a blowup criterion has been explored in Cheskidov \& Shvydkoy (2010). Because

$$
\|\boldsymbol{u}\|_{B_{\infty, \infty}^{-1}} \lesssim\|\boldsymbol{u}\|_{\mathrm{BMO}^{-1}} \simeq\|\boldsymbol{\psi}\|_{\mathrm{BMO}},
$$

the motivation is more ambitious than ours. A dichotomy-type result has been obtained in this line of research; upon a possible singularity, either i) $\|\boldsymbol{u}\|_{B_{\infty, \infty}^{-1}}$ becomes unbounded, or ii) it is bounded but there is a jump (i.e. a gap of $O(\nu)$ ) in the norm near the critical time. It is not known whether $\|\boldsymbol{u}\|_{B_{\infty, \infty}^{-1}}$ becomes unbounded or not.

In a corresponding analysis in two-dimensions, the Navier-Stokes equation in the stream function was derived in Ohkitani (2008) and its applications are described in Ohkitani (2017a,c).

\subsection{Cole-Hopf transform and Feynman-Kac formula}

We consider an analogue of the Cole-Hopf transform for the NavierStokes equations, introduced component-wise in Ohkitani (2017c), by

$$
\psi_{j}=k \log \theta_{j}, \quad(j=1,2,3),
$$

with a constant $k(\neq 0)$ and derive equations for $\boldsymbol{\theta}$. See also Vanon \& Ohkitani (2018).

The derivation of the equations for $\boldsymbol{\theta}$ is straightforward, but best stated here for completeness

$$
\begin{aligned}
& \frac{\partial \psi_{j}}{\partial t}-T_{j}\left[\nabla \psi_{j}\right]-\frac{1}{2} \triangle \psi_{j}=\frac{k}{\theta_{j}} \frac{\partial \theta_{j}}{\partial t}-k^{2} T_{j}\left[\frac{\nabla \theta_{1}}{\theta_{1}}, \frac{\nabla \theta_{2}}{\theta_{2}}, \frac{\nabla \theta_{3}}{\theta_{3}}\right]-\frac{1}{2} k\left(\frac{\triangle \theta_{j}}{\theta_{j}}-\frac{\left|\nabla \theta_{j}\right|^{2}}{\theta_{j}^{2}}\right) \\
& =k\left\{\frac{1}{\theta_{j}}\left(\frac{\partial \theta_{j}}{\partial t}-\frac{1}{2} \triangle \theta_{j}\right)-\left(k T_{j}\left[\frac{\nabla \theta_{1}}{\theta_{1}}, \frac{\nabla \theta_{2}}{\theta_{2}}, \frac{\nabla \theta_{3}}{\theta_{3}}\right]-\frac{1}{2} \frac{\left|\nabla \theta_{j}\right|^{2}}{\theta_{j}^{2}}\right)\right\},
\end{aligned}
$$


where no summation over $j$ is implied. Setting the right-hand side to zero, we obtain a system of heat equations with a potential term

$$
\frac{\partial \theta_{j}}{\partial t}=\frac{1}{2} \triangle \theta_{j}+f_{j}[\boldsymbol{\theta}](\boldsymbol{x}, t) \theta_{j},
$$

where

$$
f_{j}[\boldsymbol{\theta}](\boldsymbol{x}, t) \equiv k T_{j}\left[\frac{\nabla \theta_{1}}{\theta_{1}}, \frac{\nabla \theta_{2}}{\theta_{2}}, \frac{\nabla \theta_{3}}{\theta_{3}}\right]-\frac{1}{2} \frac{\left|\nabla \theta_{j}\right|^{2}}{\theta_{j}^{2}}, \quad(j=1,2,3 .)
$$

Hereafter no summation is implicit with respect to $j$ in $f_{j}$.

Regarding the nonlinear term as forcing in the spirit of Duhamel principle, we convert (1.13) into path-integral equations by the Feynman-Kac formula

$$
\theta_{j}(\boldsymbol{x}, t)=\mathbb{E}\left[\theta_{j}\left(\boldsymbol{W}_{t}, 0\right) \exp \left(\int_{0}^{t} f_{j}[\boldsymbol{\theta}]\left(\boldsymbol{W}_{s}, s\right) d s\right)\right] .
$$

For proof, see Ohkitani (2017c). The path-integral representation (1.15) is just another way of writing down the Navier-Stokes equations. While the formula contains complicated contents, we note that it is fully explicit, with $\boldsymbol{f}[\boldsymbol{\theta}]$ defined by (1.14) and $\boldsymbol{T}] \nabla \boldsymbol{\psi}]$ by (1.10).

For convenience, we use the following notation hereafter

$$
F_{j}[\boldsymbol{\theta}]\left(\boldsymbol{W}_{t}\right) \equiv \theta_{j}\left(\boldsymbol{W}_{t}, 0\right) \exp \left(\int_{0}^{t} f_{j}[\boldsymbol{\theta}]\left(\boldsymbol{W}_{s}, s\right) d s\right) .
$$

The exponential term $f$, which corresponds to the potential $V$ in the forced Burgers equations, controls the regularity of the Navier-Stokes equations. We emphasise that a regularity condition readily follows from (1.15). Namely, we have

$$
\int_{0}^{t}\|\boldsymbol{f}[\boldsymbol{\theta}]\|_{L^{\infty}} d s<\infty, \text { for some } k(\neq 0) \Longrightarrow \text { smooth up to time } t,
$$

or, equivalently

$$
\text { blowup at time } t \Longrightarrow \int_{0}^{t}\|\boldsymbol{f}[\boldsymbol{\theta}]\|_{L^{\infty}} d s=\infty \text {, for all } k(\neq 0) \text {. }
$$

These conditions are similar to Serrin's, but slightly different because of the first term in $\boldsymbol{f}$. It was noted in Ohkitani (2017a) that if blowup takes place, it is impossible to cancel out the two unbounded integrals $\int_{0}^{t_{*}}\|\boldsymbol{u}\|_{L^{\infty}}^{2} d t=\infty$ and $\int_{0}^{t_{*}}\|\boldsymbol{T}[\nabla \boldsymbol{\psi}]\|_{L^{\infty}} d t=\infty$ so as to make $\boldsymbol{f}$ remain bounded, no matter how carefully $k$ is chosen.

Before closing this section, a brief remark on the blowup criterion is 
in order. We distinguish two possible scenarios regarding (1.12):

1) $\theta_{j} \rightarrow 0$ and therefore $\|\boldsymbol{\psi}\|_{L^{\infty}} \rightarrow \infty$. (Note that we still have $\|\boldsymbol{\psi}\|_{\text {BMO }}<$ $\infty$, if only one, or countably many zeros appear in the flow field.)

2) $\theta_{j}>0$, but becomes non-differentiable whilst $\|\boldsymbol{\psi}\|_{L^{\infty}}<\infty$.

In connection with the above open problem, if $\|\boldsymbol{\psi}\|_{\mathrm{BMO}} \rightarrow \infty$ upon singularity, uncountably many zeros in $\theta_{j}$ must appear at the time of breakdown.

\subsection{Dynamic scaling transform}

We will apply the Cole-Hopf-Feynman-Kac formula to the dynamicallyscaled version of the Navier-Stokes equations.

Invariance under dynamic scaling implies

$$
\boldsymbol{\psi}(\boldsymbol{x}, t)=\boldsymbol{\Psi}(\boldsymbol{\xi}, \tau)
$$

and it satisfies the Leray equations of the form

$$
\frac{\partial \boldsymbol{\Psi}}{\partial \tau}-\frac{1}{2} \triangle_{\boldsymbol{\xi}} \boldsymbol{\Psi}+a \boldsymbol{\xi} \cdot \nabla_{\boldsymbol{\xi}} \boldsymbol{\Psi}=\frac{3}{4 \pi} \int_{\mathbb{R}^{3}} \frac{\boldsymbol{\rho} \times\left(\nabla \times \boldsymbol{\Psi}\left(\boldsymbol{\xi}^{\prime}\right)\right) \boldsymbol{\rho} \cdot\left(\nabla \times \boldsymbol{\Psi}\left(\boldsymbol{\xi}^{\prime}\right)\right)}{|\boldsymbol{\rho}|^{5}} \mathrm{~d} \boldsymbol{\xi}^{\prime},
$$

where $\boldsymbol{\rho}=\boldsymbol{\xi}-\boldsymbol{\xi}^{\prime}$ and $\boldsymbol{\psi}(\cdot, 0)=\boldsymbol{\Psi}(\cdot, 0)$. The difference between (1.9) and (1.20) is just one drift term, which is minimal due to the critical nature of $\boldsymbol{\psi}$. Setting $\Psi_{j}=k \log \Theta_{j},(j=1,2,3)$, we obtain as above

$$
\frac{\partial \Theta_{j}}{\partial \tau}=\frac{1}{2} \triangle_{\boldsymbol{\xi}} \Theta_{j}-a \boldsymbol{\xi} \cdot \nabla_{\boldsymbol{\xi}} \Theta_{j}+f_{j}[\Theta](\boldsymbol{\xi}, \tau) \Theta_{j} .
$$

These can also be converted into a path-integral form

$$
\Theta_{j}(\boldsymbol{x}, t)=\mathbb{E}\left[\Theta_{j}\left(\boldsymbol{X}_{t}, 0\right) \exp \left(\int_{0}^{t} f_{j}[\boldsymbol{\Theta}]\left(\boldsymbol{X}_{s}, s\right) d s\right)\right],
$$

where $\boldsymbol{X}_{t}$ denotes the Ornstein-Uhlenbeck process, generated by the modified dissipative operator $\frac{1}{2} \triangle_{\boldsymbol{\xi}}-a \boldsymbol{\xi} \cdot \nabla_{\boldsymbol{\xi}}$, i.e. the Laplace operator with a drift term.

\subsubsection{Change of probability measures}

We are in a position to make a detailed comparison between the NavierStokes equations and their dynamically-scaled counterparts (the Leray equations), using path-integral representations. Such a comparison without the Feynman-Kac formula has been carried out in Ohkitani (2017b), while a comparison with the Feynman-Kac formula in Ohkitani (2017d). 
Necessary tools are taken from stochastic analysis, whose general references include Bell (2006); Malliavin \& Thalmaier (2006); Nunno, Oksendal \& Proske (2009); Sanz-Solé (2005); Shigekawa (2004); Steele (2001); Üstünel \& Zakai (2010); Üstünel (2015). See Bru \& Yor (2002) for historical remarks including the measure changing theorems.

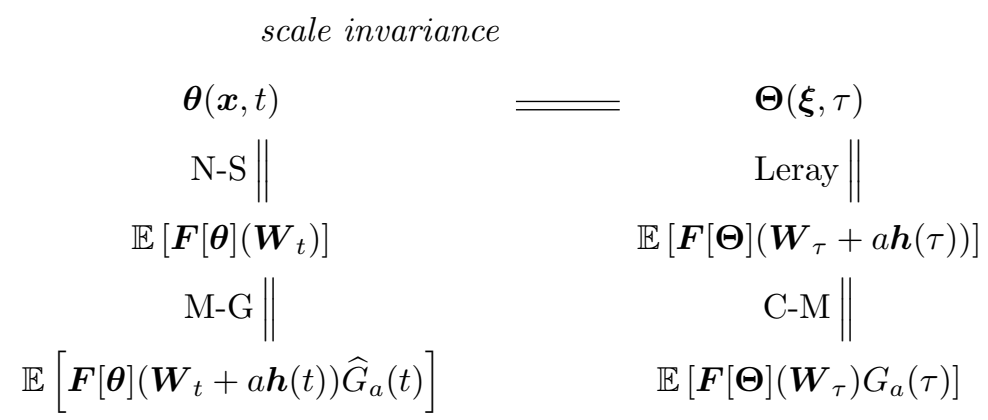

Figure 1: Scale-invariance, the dynamical equations and the transformation of probability measures; N-S stands for the Navier-Stokes equations, M-G for Maruyama-Girsanov theorem and C-M for Cameron-Martin theorem.

\subsubsection{Leray equations}

We consider the Leray equations first, because it has a global smooth solution by assumption (i.e. by construction). Defining $\Theta$ by $\Psi_{j}=$ $k \log \Theta_{j},(j=1,2,3)$ the scale-invariance becomes

$$
\boldsymbol{\theta}(\boldsymbol{x}, t)=\boldsymbol{\Theta}(\boldsymbol{\xi}, \tau) .
$$

Let us take the drift term as $\boldsymbol{b}(\boldsymbol{x})=-\boldsymbol{x}$ and $\boldsymbol{h}(t)=\int_{0}^{t} \boldsymbol{b}\left(\boldsymbol{W}_{s}\right) d s$. For a simpler comparison, we write $(\boldsymbol{x}, t)$ for $(\boldsymbol{\xi}, \tau)$. (See Figure $\mathbf{1}$ for a list of relationships with independent variables distinguished.)

The transformed variable $\boldsymbol{\Theta}$ satisfies the following equations

$$
\begin{aligned}
\boldsymbol{\Theta} & =\mathbb{E}\left[\boldsymbol{F}[\boldsymbol{\Theta}]\left(\boldsymbol{W}_{t}+a \boldsymbol{h}(t)\right)\right], \quad \text { all } t \geq 0 \\
& =\mathbb{E}\left[\boldsymbol{F}[\boldsymbol{\Theta}]\left(\boldsymbol{W}_{t}\right) G_{a}(t)\right], \quad 0 \leq t<\frac{\sqrt{2}}{a}
\end{aligned}
$$

where $G_{a}(t)$ denotes the Maruyama-Girsanov density

$$
G_{a}(t)=\exp \left(a \int_{0}^{t} \boldsymbol{b}\left(\boldsymbol{W}_{s}\right) \cdot d \boldsymbol{W}_{s}-\frac{a^{2}}{2} \int_{0}^{t}\left|\boldsymbol{b}\left(\boldsymbol{W}_{s}\right)\right|^{2} d s\right) .
$$


Here use has been made of the Cameron-Martin theorem

$$
\mathbb{E}\left[F\left(\boldsymbol{W}_{t}+\boldsymbol{h}\right)\right]=\mathbb{E}\left[F\left(\boldsymbol{W}_{t}\right) \exp \left(\int_{0}^{t} \dot{\boldsymbol{h}}(s) \cdot d \boldsymbol{W}_{s}-\frac{1}{2} \int_{0}^{t}|\dot{\boldsymbol{h}}(s)|^{2} d s\right)\right],
$$

where $F$ denotes an arbitrary functional. The time scale $\sqrt{2} / a$ has been determined by the Novikov condition for $G_{a}(t)$ to be a martingale. It is important that this time scale is larger than $1 / 2 a$, because the following comparison cannot be made otherwise.

In (1.23), "all $t \geq 0$ " means that it has a smooth solution in $t \geq 0$.

We next consider the case with finite $a$ and characterise the difference in an additive manner. Subtracting $\mathbb{E}\left[\boldsymbol{F}[\boldsymbol{\Theta}]\left(\boldsymbol{W}_{t}\right)\right]$, we have

$$
\begin{aligned}
\boldsymbol{\Theta}-\mathbb{E}\left[\boldsymbol{F}[\boldsymbol{\Theta}]\left(\boldsymbol{W}_{t}\right)\right] & =\mathbb{E}\left[\boldsymbol{F}[\boldsymbol{\Theta}]\left(\boldsymbol{W}_{t}+a \boldsymbol{h}(t)\right)-\boldsymbol{F}[\boldsymbol{\Theta}]\left(\boldsymbol{W}_{t}\right)\right] \\
& =\mathbb{E}\left[\boldsymbol{F}[\boldsymbol{\Theta}]\left(\boldsymbol{W}_{t}\right)\left(G_{a}(t)-1\right)\right] \\
& \equiv \mathbb{E}\left[\left\langle D \boldsymbol{F}[\boldsymbol{\Theta}]\left(\boldsymbol{W}_{t}+\mu \boldsymbol{h}(t)\right), a \boldsymbol{h}\right\rangle\right]
\end{aligned}
$$

which is valid for $t<\sqrt{2} / a$. Applying the usual mean-value theorem to $G_{a}(t)$ for fixed $t$, we find

$$
\frac{G_{a}-1}{a}=\left.\frac{\partial G_{a}}{\partial a}\right|_{a=\mu}, \quad \text { for some } \mu \in(0, a)
$$

where

$$
\left.\frac{\partial G_{a}}{\partial a}\right|_{a=\mu}=\left(\int_{0}^{t} \boldsymbol{b}\left(\boldsymbol{W}_{s}\right) \cdot d \boldsymbol{W}_{s}-\mu \int_{0}^{t}\left|\boldsymbol{b}\left(\boldsymbol{W}_{s}\right)\right|^{2} d s\right) G_{\mu} .
$$

The equation (1.28) can be regarded as a result of an application of "the mean-value theorem" ${ }^{1}$ to (1.26), whose precise meaning is given by (1.27). The equation (1.28) shows that the Leray equations have an extra additive term in the form of the Malliavin $H$-derivative, on top of those of the Navier-Stokes equations.

For finite $a$, we have

$$
\begin{aligned}
\boldsymbol{\Theta}-\mathbb{E}\left[\boldsymbol{F}[\boldsymbol{\Theta}]\left(\boldsymbol{W}_{t}\right)\right] & =\mathbb{E}\left[\left\langle D \boldsymbol{F}[\boldsymbol{\Theta}]\left(\boldsymbol{W}_{t}+\mu \boldsymbol{h}(t)\right), a \boldsymbol{h}\right\rangle\right] \\
& =a \mathbb{E}\left[\left.\boldsymbol{F}[\boldsymbol{\Theta}]\left(\boldsymbol{W}_{t}\right) \frac{\partial G_{a}}{\partial a}\right|_{a=\mu}\right] .
\end{aligned}
$$

We stress that the left-hand side alone defines the Navier-Stokes equations.

1 This is reminiscent of an application of the elementary mean-value theorem $f(x+a)=f(x)+a f^{\prime}(x+\mu), \quad 0<{ }^{\exists} \mu<a$. 
In passing, we note that as $a \rightarrow 0$

$$
\lim _{a \rightarrow 0}\left(\boldsymbol{\Theta}-\mathbb{E}\left[\boldsymbol{F}[\boldsymbol{\Theta}]\left(\boldsymbol{W}_{t}\right)\right]\right)=0
$$

but that

$$
\lim _{a \rightarrow 0} \frac{1}{a}\left(\boldsymbol{\Theta}-\mathbb{E}\left[\boldsymbol{F}[\boldsymbol{\Theta}]\left(\boldsymbol{W}_{t}\right)\right]\right)=\mathbb{E}\left[\left\langle D \boldsymbol{F}[\boldsymbol{\Theta}]\left(\boldsymbol{W}_{t}\right), \boldsymbol{h}\right\rangle\right] .
$$

This limit, however is not very useful as we are assuming that no finitetime blowup takes place for the Navier-Stokes equations $\left(t_{*}=1 / 2 a \rightarrow\right.$ $\infty)$.

\subsubsection{Navier-Stokes equations}

We now turn our attention to the Navier-Stokes equation of the form (1.15) and carry out an analysis in a parallel fashion. By assumption, it has a short-lived solution $\boldsymbol{\theta}$ for $t<1 / 2 a\left(=t_{*}\right)$, which satisfies

$$
\begin{aligned}
\boldsymbol{\theta} & =\mathbb{E}\left[\boldsymbol{F}[\boldsymbol{\theta}]\left(\boldsymbol{W}_{t}\right)\right], \quad 0 \leq t<\frac{1}{2 a} \\
& =\mathbb{E}\left[\boldsymbol{F}[\boldsymbol{\theta}]\left(\boldsymbol{W}_{t}+a \boldsymbol{h}(t)\right) \widehat{G}_{a}(t)\right] .
\end{aligned}
$$

Here $\widehat{G}_{a}(t)$ denotes the Maruyama-Girsanov density

$$
\widehat{G}_{a}(t)=\exp \left(-a \int_{0}^{t} \boldsymbol{b}\left(\boldsymbol{W}_{s}\right) \cdot d \boldsymbol{W}_{s}-\frac{a^{2}}{2} \int_{0}^{t}\left|\boldsymbol{b}\left(\boldsymbol{W}_{s}\right)\right|^{2} d s\right),
$$

in the Maruyama-Girsanov theorem

$\mathbb{E}\left[F\left(\boldsymbol{W}_{t}\right)\right]=\mathbb{E}\left[F\left(\boldsymbol{W}_{t}+\boldsymbol{h}\right) \exp \left(-\int_{0}^{t} \dot{\boldsymbol{h}}(s) \cdot d \boldsymbol{W}_{s}-\frac{1}{2} \int_{0}^{t}|\dot{\boldsymbol{h}}(s)|^{2} d s\right)\right]$.

As above, we have

$$
\begin{aligned}
\boldsymbol{\theta}-\mathbb{E}\left[\boldsymbol{F}[\boldsymbol{\theta}]\left(\boldsymbol{W}_{t}+a \boldsymbol{h}(t)\right)\right] & =\mathbb{E}\left[\boldsymbol{F}[\boldsymbol{\theta}]\left(\boldsymbol{W}_{t}\right)-\boldsymbol{F}[\boldsymbol{\theta}]\left(\boldsymbol{W}_{t}+a \boldsymbol{h}(t)\right)\right] \\
& =\mathbb{E}\left[\boldsymbol{F}[\boldsymbol{\theta}]\left(\boldsymbol{W}_{t}+a \boldsymbol{h}(t)\right)\left(\widehat{G}_{a}-1\right)\right] \\
& =-\mathbb{E}\left[\left\langle D \boldsymbol{F}[\boldsymbol{\theta}]\left(\boldsymbol{W}_{t}+\mu^{\prime} \boldsymbol{h}(t)\right), a \boldsymbol{h}\right\rangle\right]
\end{aligned}
$$

where

$$
\frac{\widehat{G}_{a}-1}{a}=\left.\frac{\partial \widehat{G}_{a}}{\partial a}\right|_{a=\mu^{\prime}}, \quad \text { for some } \mu^{\prime} \in(0, a)
$$


and

$$
\left.\frac{\partial \widehat{G}_{a}}{\partial a}\right|_{a=\mu^{\prime}}=-\left(\int_{0}^{t} \boldsymbol{b}\left(\boldsymbol{W}_{s}\right) \cdot d \boldsymbol{W}_{s}+\mu^{\prime} \int_{0}^{t}\left|\boldsymbol{b}\left(\boldsymbol{W}_{s}\right)\right|^{2} d s\right) \widehat{G}_{\mu^{\prime}} .
$$

For finite $a$, we find

$$
\begin{aligned}
\boldsymbol{\theta}-\mathbb{E}\left[\boldsymbol{F}[\boldsymbol{\theta}]\left(\boldsymbol{W}_{t}+a \boldsymbol{h}(t)\right)\right] & =-\mathbb{E}\left[\left(D \boldsymbol{F}[\boldsymbol{\theta}]\left(\boldsymbol{W}_{t}+\mu^{\prime} \boldsymbol{h}(t)\right), a \boldsymbol{h}\right)\right](1.35) \\
& =a \mathbb{E}\left[\left.\boldsymbol{F}[\boldsymbol{\theta}]\left(\boldsymbol{W}_{t}\right) \frac{\partial \widehat{G}_{a}}{\partial a}\right|_{a=\mu^{\prime}}\right] . \quad(1.36)
\end{aligned}
$$

Again, note that the left-hand side alone defines the Leray equations. The Navier-Stokes equations can be regarded as a perturbed version of the Leray equations.

\subsection{Summary}

In this paper we have surveyed some results on the basic issues of the Navier-Stokes equations, which have been obtained by paying attention to the scale-invariant nature of the equations.

We then show how we can generalise the concept of invariance under dynamic scaling transforms. The key step is to write down the basic equations in the dependent variables, which themselves are already scale-invariant. In three-dimensions, they are simply the vector potentials. Using dynamic scaling (as a push-forward), we obtain the Leray equations, where the dissipative operator changes from the Laplacian to the Ornstein-Uhlenbeck operator. If we move onto path-integral representations, the probability measures can be made explicit. By using the Cameron-Martin-Maruyama-Girsanov transforms (as a pull-back), we retrieve the Navier-Stokes equations modulo a Maruyama-Girsanov density $G$.

Hence it seems natural to define quasi-invariance by equivalence modulo $G$ in path integral representations. Under dynamic scaling, NavierStokes equations change their forms only slightly when written in the vector potentials. We have also seen that the difference can be interpreted in terms of the $H$-derivative.

It is of interest to study the implications of quasi-invariance on the basic issues. Now that the two equations have been shown to be very close, while the behaviour of their solutions are totally different, such a close similarity can impose constrains on the possibility of blowup. 
Particularly, it is of interest to study which specific properties of $\boldsymbol{T}[\nabla \boldsymbol{\psi}]$, if any, can make the solution to the pulled-back Navier-Stokes equations outlive the original one so that we would possibly get a contradiction.

Finally, we note that the whole arguments hold in $\mathbb{R}^{n}$ for any $n \geq 2$.

Acknowledgement This work has been supported by an EPSRC grant: $\mathrm{EP} / \mathrm{N} 022548 / 1$. 


\section{Appendix A}

\section{Wiener process}

In the triplet $(\Omega, F, \boldsymbol{P}), \Omega$ is a set of $\mathbb{R}^{d}$-valued continuous functions defined for $t \in[0, \infty), F$ the $\sigma$-algebra on $\Omega$ and $\boldsymbol{P}$ the probability measure on it.

The solution to the heat equation

$$
\frac{\partial u}{\partial t}=\frac{1}{2} \triangle u
$$

can be written

$$
u(\boldsymbol{x}, t)=\mathbb{E}\left[f\left(\boldsymbol{x}+\boldsymbol{W}_{t}\right) \mid \boldsymbol{W}_{0}=0\right],
$$

or, equivalently

$$
u(\boldsymbol{x}, t)=\mathbb{E}\left[f\left(\boldsymbol{W}_{t}\right) \mid \boldsymbol{W}_{0}=\boldsymbol{x}\right] .
$$

In the text, an abridged notation $u(\boldsymbol{x}, t)=\mathbb{E}\left[f\left(\boldsymbol{W}_{t}\right)\right]$ has been used throughout.

A link to the Gaussian probability measure $p(\boldsymbol{x}, \boldsymbol{y}, t)$ can be made explicit, Ikeda \& Watanabe (1988), by noting

$$
\mathbb{E}\left[f\left(\boldsymbol{x}+\boldsymbol{W}_{t}\right)\right]=\int f(\boldsymbol{y}) \mathbb{E}\left[\delta_{\boldsymbol{y}}\left(\boldsymbol{x}+\boldsymbol{W}_{t}\right)\right] d \boldsymbol{y},
$$

that is,

$$
p(\boldsymbol{x}, \boldsymbol{y}, t)=\mathbb{E}\left[\delta \boldsymbol{y}\left(\boldsymbol{x}+\boldsymbol{W}_{t}\right)\right]=\frac{1}{(2 \pi t)^{d / 2}} \exp \left(-\frac{|\boldsymbol{x}-\boldsymbol{y}|^{2}}{2 t}\right) .
$$

Here $\delta \boldsymbol{y}(\boldsymbol{x})$ denotes Dirac mass supported at $\boldsymbol{y}$. 


\section{Appendix B \\ Feynman-Kac formula for time-dependent potential}

For given $f_{j}(\boldsymbol{x}, t), \quad j=1,2,3$ a number of different representations are available for the (unique) solution to (1.13). To distinguish them properly, we assume here that Brownian motion starts from the origin $\boldsymbol{W}_{0}=0$, as opposed to the assumption $\boldsymbol{W}_{0}=\boldsymbol{x}$ in the main text. (Here no summation is implied on $j$.)

The expression (1.15)

$$
\theta_{j}(\boldsymbol{x}, t)=\mathbb{E}\left[\theta_{j}\left(\boldsymbol{x}+\boldsymbol{W}_{t}, 0\right) \exp \left(\int_{0}^{t} f_{j}\left(\boldsymbol{x}+\boldsymbol{W}_{s}, s\right) d s\right)\right]
$$

can be obtained by applying the time-dependent Trotter formula, see Section 11.2 of Taylor (1996).

Another form

$$
\theta_{j}(\boldsymbol{x}, t)=\mathbb{E}\left[\theta_{j}\left(\boldsymbol{x}+\boldsymbol{W}_{t}, 0\right) \exp \left(\int_{0}^{t} f_{j}\left(\boldsymbol{x}+\boldsymbol{W}_{s}, t-s\right) d s\right)\right]
$$

can be found in Freidlin (1985).

Yet another form

$$
\theta_{j}(\boldsymbol{x}, t)=\mathbb{E}\left[\theta_{j}\left(\boldsymbol{x}+\boldsymbol{W}_{t}, 0\right) \exp \left(\int_{0}^{t} f_{j}\left(\boldsymbol{x}+\boldsymbol{W}_{t}-\boldsymbol{W}_{s}, s\right) d s\right)\right]
$$

can be found in Friedrichs et al. (1957). The expression (B.3) can be extended to the case where the potential term $f_{j}$ itself is stochastic Chow (2014).

We can make use of the alternative forms of functional integrals by changing the all arguments in $f_{j}(\cdot, \cdot)$ accordingly. 


\section{References}

Bell, D. R. (2006) The Malliavin Calculus. Dover, New York.

Bru, B. \& Yor, M. (2002) Comments on the life and mathematical legacy of Wolfgang Doeblin. Finance and Stochastics, 6, 3-47.

Chae, D. (2007) Nonexistence of asymptotically self-similar singularities in the Euler and the Navier-Stokes equations. Math. Ann., 338, 435-449.

Cheskidov, A. \& Shvydkoy, R. (2010) The Regularity of Weak Solutions of the 3D Navier-Stokes Equations in $B_{\infty, \infty}^{-1}$. Arch. Rat. Mech. Anal., 195, 159-169.

Chow, P.L. (2014) Stochastic Partial Differential Equations. CRC Press, Boca Raton.

Cole, J. D. (1951) On a linear quasilinear parabolic equation in aerodynamics. Q. Appl. Math., 9, 225-236.

Cole, J. D. (1949) Problems in transonic flow. PhD Thesis, California Institute of Technology (pages 56 and onward).

Constantin, P. \& Foias, C. (1988) Navier-Stokes Equations. University of Chicago Press, Chicago.

Doering, C. R. (2009) The 3D Navier-Stokes problem. Annu. Rev. Fluid Mech., 41 109-128.

Doering, C.R. \& Gibbon, J.D. (1995) Applied Analysis of the Navier-Stokes Equations. Cambridge University Press, Cambridge.

Escauriaza, L., Seregin, G. \& Sverak, V. (2003) $L_{3, \infty}$-solutions of the NavierStokes equations and backward uniqueness. Russ. Math. Surv., 58, 211250 .

Freidlin, M.I. (1985) Functional Integration and Partial Differential Equations Annals of Mathematics Studies 109, Princeton University Press, Princeton.

Friedrichs, K.O., Seidman, T., Wendroff, B., Shapiro, H.N., \& Schwartz, J. (1957) Integration of functionals New York University, Institute of Mathematical Sciences.

Hopf, E. (1950) The partial differential equation $u_{t}+u u_{x}=\mu u_{x x}$. Commun. Pure Appl. Math., 3, 201-230. 
Hou, T. \& Li, R. (2007) Nonexistence of locally self-similar blow-up for the 3D incompressible Navier-Stokes equations. Dis. Cont. Dyn. Sys., 18, $637-642$

Ikeda, I. \& Watanabe, S. (1988) Stochastic Differential Equations and Diffusion Processes North Holland, Amsterdam.

Kato, T. \& Fujita, H. (1962) On the nonstationary Navier-Stokes system. Rendiconti del Seminario Matematico della Universitá di Padova, 32, 243-260.

Koch, K. \& Tataru, D. (2001) Well-posedness for the NavierStokes equations. Adv. Math., 157, 22-35.

Leray, J. (1934) Essai sur le mouvement d'un liquide visqueux emplissant l'espace. Acta Math., 63, 193-248.

Malliavin, P. \& Thalmaier, A. (2006) Stochastic Calculus of Variations in Mathematical Finance. Springer, Berlin, Heidelberg.

Nečas, J., Růžička, M. \& Šverák, V. (1996) On Leray's self-similar solutions of the Navier-Stokes equations. Acta Math., 176, 283-294.

Nunno, G.D., Oksendal, B. \& Proske, F. (2009) Malliavin Calculus for Lévy Processes with Applications to Finance, Berlin, Springer.

Ohkitani, K. (2008) A miscellany of basic issues on incompressible fluid equations. Nonlinearity, 21, 255-271.

Ohkitani, K. (2015) Dynamical equations for the vector potential and the velocity potential in incompressible irrottational Euler flows: a refined Bernoulli theorem. Phys. Rev. E., 92, 033010.

Ohkitani, K. (2016) Study of the Navier-Stokes regularity problem with critical norms. Fluid. Dyn. Res., 48, 021401.

Ohkitani, K. (2017a) Characterization of blowup for the Navier-Stokes equations using vector potentials, AIP Advances, 7, 015211

Ohkitani, K. (2017b) Near-invariance under dynamic scaling for the NavierStokes equations in critical spaces: a probabilistic approach to regularity problems. J. Phys. A: Math. Theor., 50, 045501.

Ohkitani, K. (2017c) Analogue of the Cole-Hopf transform for the incompressible Navier-Stokes equations and its application. Journal of Turbulence, 18, 465-479.

Ohkitani, K. (2017d) Cole-Hopf-Feynman-Kac formula and quasi-invariance for Navier-Stokes equations. J. Phys. A: Math. Theor. 50, 405501.

Robinson, J. C. \& Sadowski, W. (2009) The regularity problem for the three-dimensional Navier-Stokes equations. Partial Differential Equations and Fluid Mechanics, London Math. Soc. Lec. Note Ser. 364, 185-206.

Serrin, J. (1963) The initial value problem for the Navier-Stokes equations. in Nonlinear Problems, (ed.) R.E. Langer, 69-98. University of Wisconsin Press, Madison.

Sanz-Solé, M. (2005) Malliavin Calculus with Applications to Stochastic Partial Differential Equations. EPFL Press, Lausanne.

Shigekawa, I. (2004) Stochastic Analysis. American Mathematical Society, Province. 
Steele, J.M. (2001) Stochastic Calculus and Financial Applications. Springer, New York.

Taylor, M. (1996) Partial Differential Equations II: Qualitative Studies of Linear Equations. Springer, New York.

Üstünel, A.S. (2015) Analysis on Wiener Space and Applications. arXiv preprint arXiv:1003.1649.

Üstünel, A.S. \& Zakai, M. (2010) Transformation of Measure on Wiener Space. Springer, Berlin Heidelberg.

Vanon, R. \& Ohkitani, K. (2018) Applications of a Cole-Hopf transform to the 3D Navier-Stokes equations. Journal of Turbulence, $19,322-333$. 